\title{
Modeling of cartographic parameters in the flow part of an energy machine for safe vibro-drying of grain
}

\author{
Vasiliy Vinogradov ${ }^{1, *}$, Radik Gibadullin ${ }^{2}$ and Anvar Gumerov ${ }^{1}$ \\ ${ }^{1}$ Kazan National Research Technical University named after A.N. Tupolev, 420111 Kazan, Russia \\ ${ }^{2}$ Kazan State Agrarian University, 420015 Kazan, Russia
}

\begin{abstract}
The present article is devoted to the modeling of a gas-air flow at the nozzle of the energy machine designed for the use in the agricultural sector. The machine has an option of secure vibro-drying of grain crops and their control, which increases the drying performance both in the steady-state and transient modes. The article also presents a mathematical model describing acoustic processes in a flow part of the energy machine and suggests the ways to improve them for their safe operation. The authors outline the principles of construction of complex drying systems of aero-acoustic cartography, which allow controlling the parameters of energy machines serving grain crops drying and the climatic parameters depending on the volume of grain dryers. This is very important for productivity increase and automated control in real time, supplemented by methods and means of measurements of gas-dynamic parameters in the flow and at the nozzle of the energy machine. These methods are aimed at increasing information capacity and the level of algorithmic non-destructive condition monitoring of rotor blades as well as providing data in terms of parametric and structural uncertainties of the gas-air flow as controlled by the internal cross-section of the flow part, and the output section of the energy machine. Statistical approaches are used to determine the coordinates of sensors and the recovery error on the example of a model problem for monitoring the radiation field created by a continuous monopole emitter. The possibility of using fiber technologies for vibro-drying of grain is a new contribution to the improvement and effective management of grain drying process.
\end{abstract}

\section{Introduction}

As shown by the studies in the review article, combination of all sub-methods characterized by multifaceted tasks into a single aero-acoustic space [1-8] is one of the most effective ways to improve metrological characteristics and extend the functionality of acoustic (noise) control method parameters of the gas flow in the flowing part of the energy machine. This allows controlling the parameters of both the field, which is the volume of the drying chamber, and the field of acoustic impact of malfunctions accompanying operation of energy machines in different climatic conditions.

One of the problems that can be solved by using this combination is the problem of generating sound by a gas flow at low Mach numbers when controlling the turbine blades of the flow part of an energy machine used in agriculture. This article is devoted to modeling of gas-air flow on the section of the turbo-machine nozzle for steady and non-steady operation modes, as well as to the development and research of distributed fiber systems for aeroacoustic control of gas-air flow parameters at the nozzle of the energy machine. The possibility of using fiber technologies for vibro-drying of grain is a new contribution to the improvement and effective management of grain drying process. In practice, monitoring and measurement of spatial distributions of acoustic fields based on fiber-optic sensors of various physical nature are carried out by registering signals from sensors placed in a set of control points. In the present research, such control systems are referred to as multipoint, although they may consist of a single sensor that is moved around the control points.

Let us consider the problem of placing control points and the problem of restoring the spatial distribution of the field in dimensions for a finite set of points separately. Distributed fiber-optic sensors provide many advantages such as high sensitivity to changes in physical fields and insensitivity to electromagnetic interference, remote sensing capability, low cost and size. They can also be used in aggressive environments. These advantages give grounds for the use of optical fiber as a control sensor for aeroacoustic measurements at the output of an energy machine.

\section{Objects and methods of research}

The current stage of development of energy machines is characterized by the fact that along with the requirements for improving their specific characteristics, the requirements for safety, reliability and durability of

* Corresponding author: vawin@mail.ru 
their operation continue to increase. Today, a new system of operation by technical condition is being implemented in the practice of operation of turbo machines, which allows for more effective use of the individual technical capabilities of each specific product. Successful implementation of the system requires such methods and control means that would allow detecting malfunctions at an early stage. This would reduce environmental and economic costs of operation and repair, and ensure more intense development of resource capabilities of energy machines.

Now, defects in the flow part of energy machines such as burnouts breaks in the turbine blades as well as other accompanying defects of the combustion chamber (deposits of carbon) are detected in operation only during periodic inspections with endoscopes, although the development of the defect from the origin to almost complete burn - out of one or more blades occurs in a very short period of time - from a few minutes to several hours. The main detector of such defects is the gas flow that washes the elements of energy machines. Existing means of continuous monitoring (vibration sensors or temperature sensors behind the turbine) do not allow detecting this defect due to its locality and due to the fact that defects in fixed parts have negligible impact on the vibration characteristics of an energy machine as a whole.

At the same time, defects in moving parts can be detected by vibration sensors. The number of measured standard parameters is not sufficient for an effective sensitive diagnostics of energy machines, and the installation of additional sensors is problematic due to the weight restriction and necessary modifications [912]. Based on the above, search for such defects and detection of their signs must be carried out on the basis of a detailed study of the gas flow by acoustic and gas dynamic parameters at the nozzle during cold scrolling, which is a rotor run-out or purge from an independent source in the form of a reference probing flow (Fig. 1).

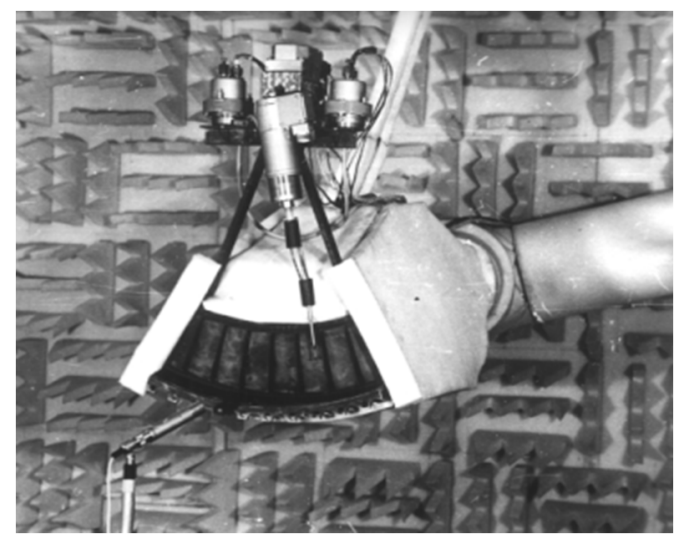

Fig. 1. Setting a microphone behind the blades of the energy machine on the stand

Accordingly, the choice of these two methods to assess their further development has required analyzing the mathematical models of air flows of energy machines and their influence. The problem is covered by many authors. For further analysis, we have selected the Lighthill acoustic analogy having similar description of the physical processes of mathematical simulation of the unperturbed and perturbed flows at the nozzle for different parameters of the jet flow based on experimental data. Information technology for building a mathematical model of a dynamic object based on experimental data fully reflects physics in terms of detecting malfunctions at an early stage.

\subsection{Mathematical model of undisturbed flow of the flow part of an energy machine}

The operation of an energy machine on a cold start is a mixed process, both non-established and established. The state of the turbo machine at each moment of time can be uniquely described by $m+1$ of the state variable $\mathrm{X} 0 \mathrm{X} 1 \mathrm{X} 2 \ldots \ldots . \mathrm{Xm}$. When conducting an experiment on an energy machine, the initial values of state variables are set at time $t 0$, and the current values of state variables are measured at $N+1$ point $t_{0}, t_{1}, t_{2}, \ldots, t_{N}$ belonging to the time interval $\left[t_{0}, t_{N}\right]$. Let the state of the turbo machine at each moment of time be uniquely described by $m+1$ of the state variable $\mathrm{X}_{0} X_{1} X_{2} \ldots \ldots . X_{m}$. The initial values of state variables are set at time $t_{0}$, and the current values are measured at $\mathrm{N}+1$ point $t_{0}, t_{1}, t_{2}, . ., t_{N}$ belonging to the time interval $\left[t_{0}, t_{N}\right]$. The coefficients in each equation are found from the minimum condition of the standard approximation. For example, for the $i$-th equation, $(i=0,1, \ldots, m)$ coefficients are found from the minimum condition of the function.

$$
\left\{\begin{array}{l}
\frac{d X_{0}}{d t}=a_{00} f_{0}(t)+a_{01} f_{1}(t)+\ldots+a_{0 k} f_{k}(t), \\
\cdots \ldots \ldots \ldots \ldots \ldots \ldots \ldots \ldots \ldots \ldots \ldots \ldots \ldots \ldots \ldots \ldots \ldots \ldots \ldots \ldots \\
\frac{d X_{m}}{d t}=a_{m 0} f_{0}(t)+a_{m 1} f_{1}(t)+\ldots+a_{m k} f_{k}(t)
\end{array}\right.
$$

When building a mathematical model of the undisturbed flow of the flow part of the energy machine, the rotation frequency $N(\mathrm{r} / \mathrm{min})$, thrust $R(\mathrm{~kg} / \mathrm{s})$ and sound pressure level $L(\mathrm{Db})$ were used as state variables, the experimental values of which were measured on a laboratory stand. The mathematical model of the energy machine is developed on the basis of experimental data in such a way that it is possible to predict the operation of a dynamic system with or without taking into account external factors, depending on the problem statement.

Experimental values used to build a mathematical model and having a small error over a certain time indicate that the model is adequate, and that the process of building a mathematical model of the energy machine flow is considered complete.

However, external disturbance to the measured values at initial changes in parameters leads to the change in calculated parameters, and the sound pressure levels will not exceed the permissible value. Then the difference $\triangle$ between the measured value of the parameter and the calculated one $\triangle$ will increase over time and thus will show negative impact of an external factor on individual parts of the turbo machine body, taking into account all 
the interdependent parameters laid down in the mathematical model [13-18].

The problem of localization of acoustic sources in 2D and $3 \mathrm{D}$ data measurement formats for improving the efficiency of early diagnostics of internal defects of energy machines has been solved. The point $(x 0, y 0)$ is the location of an acoustic source of a narrow-band signal, which is detected by sensors $D 1 \ldots . D n$. To find the coordinates of the acoustic radiation source $(x 0, \ldots y 0)$, a system of nonlinear equations has been developed:

$$
\left\{\begin{array}{l}
\alpha_{2}^{2}\left(x_{0}^{2}+y_{0}^{2}\right)=\alpha_{1}^{2}\left[(x-d)^{2}\right]+y_{0}^{2} \\
\alpha_{3}^{2}\left(x_{0}^{2}+y_{0}^{2}\right)=\alpha_{1}^{2}\left[\left(2 d-x_{1}\right)^{2}\right]+y_{0}^{2}
\end{array}\right\}
$$

Based on the above, it has been found that the aeroacoustic control method based on fiber-optic technologies allows taking a new step towards improving the safety of turbo machines during their operation in different climatic conditions.

\section{Discussion and analysis of results}

To improve the control of technical condition of the working blades of the flow part of the operated energy machine, various tools and methods are used for optimizing the distributed fiber cartographic aeroacoustic system, which allows for timely detection of malfunctions. Due to the complexity of the machine design, the control can be surface, internal and external. New monitoring tools are being developed for this purpose: thermocouples, microphones, hydrophones, vibration sensors, combined sensors and signal processing equipment. For our case of acoustic monitoring, we will focus on some sensors that are necessary for high-quality acoustic signal reception.

Intense development and implementation of fiberoptic telecommunications systems have led to the emergence of one of the most dynamically developing areas of optoelectronics — fiber-optic sensors of physical quantities controlled in agricultural machinery used in foreign countries. We have proposed the installation of fiber-optic sensors depending on the type of controlled space or the output section of an energy machine nozzle (Fig. 2).

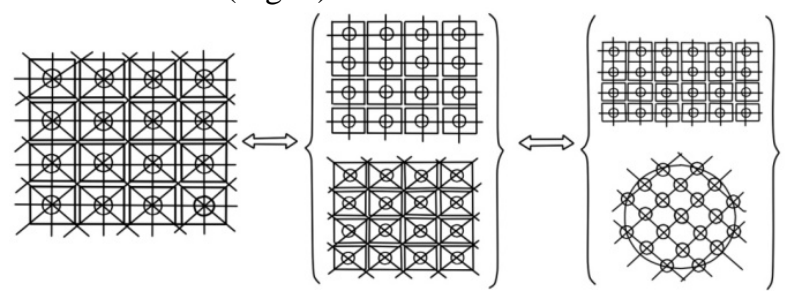

Fig. 2. Scheme of measuring lines layout

The scheme shown in Fig. 2 is characterized by a "large angle", i.e. the sufficiency of the number of directions for observations. The task of restoring the functions of physical fields using fiber-optic sensors can be divided into several stages: sensor placement, sampling, receiving, processing projection data, and reverse projection [19-20].

The algorithm for determining the spatial placement of control points and restoring the acoustic field from discrete counts is based on a priori information about the statistics of the fields to be restored. Since each aeroacoustic system of turbo machine parameters control is developed for all types of turbo machines, in real situations that are not amenable to analytical calculation, an experiment can be organized to recreate the operating modes of acoustic field excitation and measure the set of acoustic field $T(\vec{x})$ implementations using a universal measuring system with a large number of sensors. Then, the necessary statistical characteristics are calculated: mathematical expectations $\vec{m}$ and the correlation matrix $R$. The use of an iterative algorithm for determining the coordinates of aeroacoustic control points consists in sequentially supplementing the existing set of control points with a new control point, the position of which corresponds to the maximum norm $\left\|\vec{\eta}_{k}^{\perp}\right\|^{2}$ of the vector $\vec{\eta}_{k}$ orthogonalized with respect to the vectors selected in the previous steps $\vec{\eta}_{p}^{\perp}, p=1,2, \ldots, k-1$. The set of control points presented in this way corresponds to the maximum value of the determinant of the correlation matrix of the values of the random acoustic field at the control points, reducing the values of non-diagonal elements of the correlation matrix by modulus in comparison with the diagonal elements. This algorithm for determining the coordinates of control points is more computationally economical.

\section{Recommendations}

However, it is possible to use the available a priori information about the restoration of a cartographic acoustic field from measurements of its finite number of points much more widely if all $I$ coefficients are determined from the optimization condition by some criterion. It is possible to determine the spatial correlation of atmospheric phenomena that very accurately describe the effects on the cultivation of crops, as well as on climate changes and the impact on surface defects of agricultural machinery used in different operating conditions.

It should be noted once again that the algorithms for determining the spatial placement of control points and restoring the acoustic field from discrete counts are based on a priori information about the statistics of the fields to be restored. Since each aeroacoustic system of turbo machine parameters control is developed for all types of turbo machines, in real situations that are not amenable to analytical calculation, an experiment should be carried out to recreate the operating modes of acoustic field excitation and measure the set of acoustic field $T(\vec{x})$ implementations using a universal measuring system with a large number of sensors. Then, the 
necessary statistical characteristics are calculated: mathematical expectations and the correlation matrix.

\section{5ロ\&onclusion}

The mathematical model of the gas-air flow at the nozzle of the energy machine opens up opportunities to serve as a new add-on for creating a new improved complex model that includes dozens of interdependent parameters, which will reduce the time of monitoring and diagnostics, especially in the cold scroll mode. The mathematical model lets determine the ways to predict the behavior of dependent parameters over a certain period of time.

Based on the statistical approach, algorithms for monitoring and restoring acoustic fields in the nozzle plane of the energy machine have been developed and implemented as computational procedures.

The effectiveness of the proposed algorithms for monitoring and restoring fields is confirmed by the results of mathematical modeling of the radiation field control system. A system of automatic control of gas-air flow parameters at the turbo machine nozzle in the form of various measuring control lines depending on the shape of the controlled objects has been proposed.

The problems of placing control points and the problem of restoring the spatial distribution of the field in dimensions for a finite set of points separately have been studied. Restoration of the acoustic field of energy machines is implemented based on measurements at the output of fiber-optic sensors, using only a statistical approach. Restoration of the noise field is represented as continuous functions of spatial coordinates based on measurement results in a discrete set of points with a priori information about the properties of the measured noise fields.

The process of restoration of the energy machine's acoustic field has been implemented according to the data of measurements. Field reconstruction is based on the results of measurement in a discrete set of points with a priori information about the properties of the measured acoustic fields.

The possibility of using fiber technologies using the automatic control system for grain drying is a new contribution to the efficient operation of energy machines based on continuous monitoring of grain material in real time.

\section{References}

1. R. Gibadullin, A. Gubeidullina, S. Glushko, G. Petrova, V. Vinogradov, The dioxin regulation criteria in the agro-industrial complex of Russia, BIO Web Conf., 17 (2020)

2. O.B. Vitrik, The problem of "sensitive skin" and fiber-optical measuring systems, Sorosov ed. J., 7(1), 108-115 (2001)

3. S.A. Petukhov, L.S. Kurmanova, M.P. Erzamaev, D.S. Sazonov, D.S. Chinchenko, Improving the environmental safety of transport engines when using modified engine oil, Ecol. and indust. of Russ., 24(1), 9-13 (2020) Retrieved from: https://www.elibrary.ru/author_items.asp?authorid= 539666https://www.elibrary.ru/author_items.asp?aut horid $=539665$

4. E.N. Proshkin, V.I. Kurdyumov, O.M. Kanyaeva, Justification of parameters of contact seed dryer with a vibrational transporting working body, Bulletin of Ulyanovsk State Agricultural Academy, 4(40), 193-198 (2017)

5. V.I. Kurdyumov, Yu.M. Isaev, A.A. Pavlushin, S.A. Sutyagin, Peculiarities of grain movement on dryer spiral disc, Bulletin of Ulyanovsk State Agricult. Acad., 1(45), 12-17 (2019)

6. M.A. Mastepanenko, S.Z. Gabriyelyan, I.N. Vorotnikov, S.V. Mashkov, Methods for determining the informative parameters when processing the measuring signals transducers. capacitive transducers, Res. J. of Pharmac., Biolog. and Chem. Sci., 9(6), 1846-1851 (2018)

7. E.N. Gottlich, A Method for Overall Condition Monitoring by Controlling the Efficiency and Vibration Level of Rotating Machinery, Proc. of the Institut. of Mechan. Engineers - Vibrat. in Rotat. Machin., 445-447 (1988)

8. J.W. Hill, N.C Baines, Application of an Expert System to Rotating Machinery Health Monitoring, Proc. of the Institut. of Mechan. Engineers - Vibrat. in Rotat. Machin., 449-454 (1988)

9. A. Van Dan Bos, Alternative interpretation of maximum entropy stectal analysis, IEEE Trans. Inform. Theory, IT-17, 493-494 (1971)

10. T.Yu. Gumerov, G.L. Norina, L. Gabdukaeva, Assessment of risk associated with drinking water with respect to indicators of olfactory and reflex effect, IOP Conf. Ser. Mater. Sci. and Engineer., 687(6), 27-33 (2019)

11. J.M. Senior, S.E. Moss, S.D. Cusworth, Multiplexing Techniques for Non-interferometric Optical Point-Sensor Networks, Fiber and Integr. Opt., 17, 3-20 (1998)

12. A. Michailova, E.E. Zinurova, T.Yu. Gumerov, O. Reshetnik, Prevention of health hazards while working with chromium compounds, Helix, 9(5), 5454-5457 (2019)

13. Yu.I. Choni, V.I. Anfinogentov, Statistical approach to problems of synthesis in multipoint measurement system fields, Cybern. and comput. Engineer., 79, 82-87 (1988)

14. Yu.N. Kulchin, Z.V. Zakasovskaya, Uneven laying schemes of measuring lines in distributed fiber-optic systems, Inform. and control syst., 3, 61-71 (2009)

15. V.I. Anfinogentov, Mathematical modeling of microwave heating of dielectrics (Doct. Dissertation) (Kazan, 2006), 340 p.

16. I.V. Ivshin, S.Yu. Garmonov, V.N. Gavrilov, M.E. Shirokov, Vibroacoustic method for 
determining the technical condition of gears, Bull. of the Kazan Technol. Univer., 2, 120-124 (2009)

17. I.V. Ivshin, Development of a vibroacoustic method for determining the technical condition of products of complex shape using the results of numerical modeling, Bull. of Kazan Technol. Univer., 2, 125-128 (2009)

18. A.Zh. Sakhabutdinov, Procedure for sampling dual address fiber Bragg structures as sensors of a radiophoton low-sensory system, Phys. of wave process., 21(3), 101-109 (2007)
19. G.A. Morozov, N.E. Stakhova, I.P. Talanov, P.I. Talanov, A.V. Stepura, Use of electromagnetic fields of extremely high frequency band for productivity improvement of cereal crops, in: Int. Conf. on Antenna Theory and Techniques: Dedicated to 95 Year Jubilee of Prof. Ya.S. Shifrin, ICATT - Proceedings, 7136889 (2015)

20. A.V. Morozov, G.D. Fedotov, D.R. Musharapov, Results of bench tests for wear resistance of mobile straight-sided spline fittings after electromechanical hardening, Bulletin of Ulyanovsk State Agricult. Acad., 2(46), 19-23 (2019) 